Orthopäde $2009 \cdot 38: 113$

DOI 10.1007/s00132-008-1360-z

Online publiziert: 5. Februar 2009

(c) Springer Medizin Verlag 2009

\author{
C. Carstens \\ Galenus-Klinik, Fachklinik für Wirbelsäulenchirurgie, Stuttgart
}

\title{
Idiopathische Skoliose
}

eine kürzere Spondylodesestrecke gerechtfertigt ist und ob sich hieraus eine höhere Patientenzufriedenheit mit dem Operationsresultat ergibt, bleibt jedoch abzuwarten.

Bei welchem Skoliosentyp ist primär eine ventrale und bei welchem primär eine dorsale Korrektur zu bevorzugen?

Als nach wie vor völlig unbefriedigend gelöst ist das Problem der Behandlung von infantilen und juvenilen Skoliosen anzusehen. Die äußere Fixation durch Korsette gilt zwar nach wie vor als Standard, stößt jedoch immer wieder auf Akzeptanzschwierigkeiten. Reicht evtl. eine geringere Tragezeit aus, um den gleichen Effekt zu erzielen? Oder stellt die theoretisch faszinierende Vorstellung einer inneren Fixation durch ein dynamisches, mitwachsendes Instrumentarium schon heute eine echte Alternative dar?

Schon vor vielen Jahren hat Dubousset sich mit der Frage auseinandergesetzt, ob die Entwicklung einer idiopathischen Skoliose u. U. aus einer Störung des Melatoninstoffwechsels resultiert und ob sich aus dieser Überlegung therapeutische Ansätze ergeben, sodass in ferner Zukunft vielleicht ein Medikament ein Korsett ersetzen kann. Diese Hoffnungen haben sich zwar nicht bestätigt, aber die Grundlagenforschung hat weiter daran gearbeitet, den Begriff „idiopathische“ Skoliose weiter zu differenzieren. Hoffnungsvolle Ansätze ergeben sich auf molekularbiologischer Basis bei dem Versuch, die potentiell progredienten - und damit behandlungspflichtigen - von den benignen, nichtprogredienten Skoliosen abzugrenzen. Wird in naher Zukunft zur Beantwortung dieser Frage ein einfacher Bluttest ausreichend sein?

Vor völlig anderen Herausforderungen steht man bei der Behandlung der Skoliose des älteren Menschen.
Und zu guter Letzt erhebt sich die Frage, wie diese $z$. T. sehr aufwändigen Therapieverfahren unter den Bedingungen des DRG-Systems so finanzierbar sind, dass alle Patienten, die einer solchen Behandlung bedürfen, auch davon profitieren können.

Aus den oben stehenden Überlegungen folgt, dass sich auf dem Gebiet der „idiopathischen Skoliose" in den letzten Jahren eine solche Fülle von neuen Entwicklungen, Trends und Perspektiven aber auch Fragen ergeben haben, dass „Der Orthopäde" sich in einem speziellen Themenheft mit dem gegenwärtigen Stand der Erkenntnisse und der Diskussion auseinandersetzt.

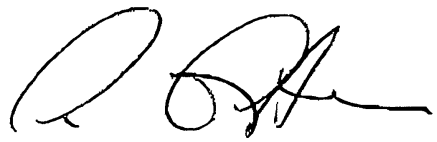

C. Carstens

\section{Korrespondenzadresse}

Prof. Dr. C. Carstens

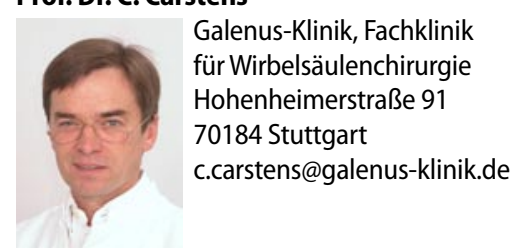

